\title{
Dix années d'hydrologie sur le Rhône
}

\section{Ten years of hydrology on the Rhône}

\author{
PAR M. HENRY \\ INGÉNIEUR EN CHEF DES PONTS ET CHAUSSÉES HORS CADRE, \\ DIRECTEUR DE LA CIE NATIONALE DU RHONE, CHARGÉ DES SERVICES D'ETULES \\ ET M. MAUCHAMP

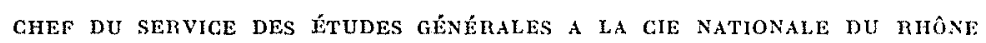

\begin{abstract}
Avec la mise en chantier de l'aménagement de Donzère-Mondragon sur le Rhône d̀ l'aval de Lyon, la Compagnie Nationale du Rhône a été amenée d̀ préciser les caractéristiques hydrométriques du fleuve.

A cet effet, un ensemble d'installations en vule d'observations et de mesures ont été établies. L'objet de la communication est de présenter les résultats obtenus et d'en dégager les enseignements pour l'étude d'un grand bassin aux données multiples.
\end{abstract}

Jusqu'en 1941, les travaux de la Compagnie Nationale du Rhône furent localisés dans le secteur du Haut-Rhône compris entre la frontière franco-suisse et Seyssel en vue de l'exécution de Génissiat.

Lor'squ'en 1941 les études relatives au BasRhône furent entreprises, plus spécialement cel-

\begin{abstract}
Before starting construction work on the Donzire-Mondragon project downstream of Lyons, the Compagnie Nationale du Rhone had to determine the hydraulic characteristics of the Rhone.

A system of hydrological observation and measurement stations was set up for this purpose.

The aim of this paper is to present the results that were obtained and to show what pointers they can give for the study of large basins affected by many factors.
\end{abstract}

les de l'aménagement de Donzère-Mondragon sur le tiers central compris entre l'Isère et l'Ardèche, les connaissances hydrologiques se révélèrent rapidement insuffisantes.

Notre action ne devint vraiment efficace que vers 1949 , d'où le titre de cette communication :

«Dix ans d'hydrologie sur le Rhône».

\section{I. - SITUATION SUR LE RHONE AU DÉPART}

Vous avez marqué une certaine surprise de m'entendre dire que les connaissances hydrologiques sur le Rhône s'étaient révélées insuffisantes. Vous pensiez à ce travail de bénédictin, que chacun connaît, effectué par M. Pardé sur son fleuve de prédilection: le Rhône.

Nous avons trop puisé à cette source intarissable de renseignements pour la méconnaitre.

Nous connaissons aussi l'ossature remarquable que le Service Spécial du Rhône avait ins- tallée tout au long du cours du fleuve avec des échelles réparties tous les 250 à 300 mètres.

Mais, pour les uns et les autres, les problèmes posés n'étaient pas les mêmes:

-- Nos besoins d'ingénieurs imposaient plus de précision, surtout quant aux débits, et M. Pardé, que nous avons vu « souffrir » sur la détermination d'un module ou d'un débit de crue, qui n'a jamais hésité, avec une singulière bonne foi, à amender ses propres 
chiffres, ne me contredira pas sur l'imprécision relative des connaissances de l'époque. Le Service spécial du Rhône se préoccupait surtout de suivre l'évolution de seuils locaux pour la navigation à courant libre; il pouvait se permettre de travailler par section dans le relatif sans référence imposée au critère absolu des débits.

Ainsi, et malgré l'intérêt - pour ne pas dire la passion - que beaucoup ont porté pour le Rhône, - en 1941 — à l'ère de la mécanique deux limnigraphes seulement étaient en fonctionnement sur le cours du fleuve à l'aval de Lyon.

Du point de vue des débits, il avait été procédé à des jaugeages au flotteur, quelques-uns à l'hydrotachymètre et à des essais très limités de mesures au moulinet.

\section{II. - LES INSTALLATIONS REALISEES}

Notre premier souci fut donc d'installer des limnigraphes et des stations de jaugeage, tant d'ailleurs sur le Haut-Rhône que sur le BasRhône.

\section{a) Limnigraphes (carte 1).}

En 1949, nous avions établi ou remis en service quatre stations limnigraphiques seulement: Sault-Brénaz et Anthon sur, le Haut-Rhône, Joviac (Le Teil) et Saint-Etienne-des-Sorts pour encadrer la chute de Donzère-Mondragon.

La cadence devait alors s'accélérer et, à l'heure actuelle, nous exploitons 23 limnigraphes sur le Rhône et 9 sur les affluents, sans compter les appareils qui sont installés sur les aménagements hydro-électriques pour leur exploitation.

En principe, un limnigraphe est installè à l'aval du confluent de chaque affluent important ce qui permet de suivre l'influence de cet affluent sur le régime du fleuve.

Ces installations ne présentent pas de caractère particulier, sauf qu'en deux emplacements nous avons installé un type de limnigraphe « incliné » pour lequel le flotteur glisse dans une gaine inclinée.

Le fruit de notre expérience pour l'exploitation des limnigraphes se résume ainsi :

- l'automaticité de l'appareil ne doit pas empê. cher de faire effectuer autant que possible un contrôle journalier;

- lobservateur doit tenir une fiche de contrôle portant à la fois les cotes lues sur l'appareil et à l'échelle de référence et les heures lues sur l'appareil et la montre;

- le réglage de l'appareil ne doit pas se faire chaque jour, mais seulement en fin de semaine lors de la pose de la nouvelle feuille; la fiche de contrôle permettra de procéder aux corrections éventuelles à apporter à l'enregistrement. b) Stations de Jaugeages (carte 1).

Le choix de leur emplacement est encore plus délicat et il faut se prémunir contre la tentation d'utiliser une installation existante, par exemple un bac, qui ne donne que très rarement satisfaction.

\section{(1) Stations limnigraphiques_C.N.R. (-'Stations de jaugeages}

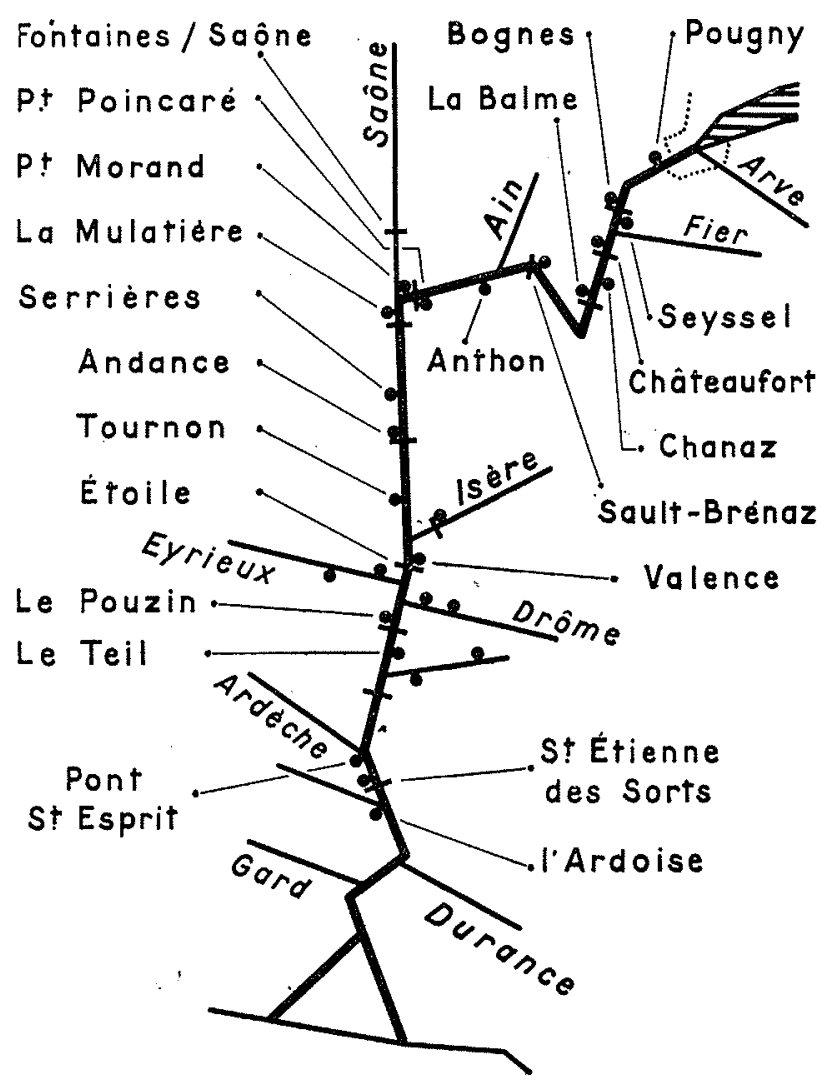

FiG. 1 


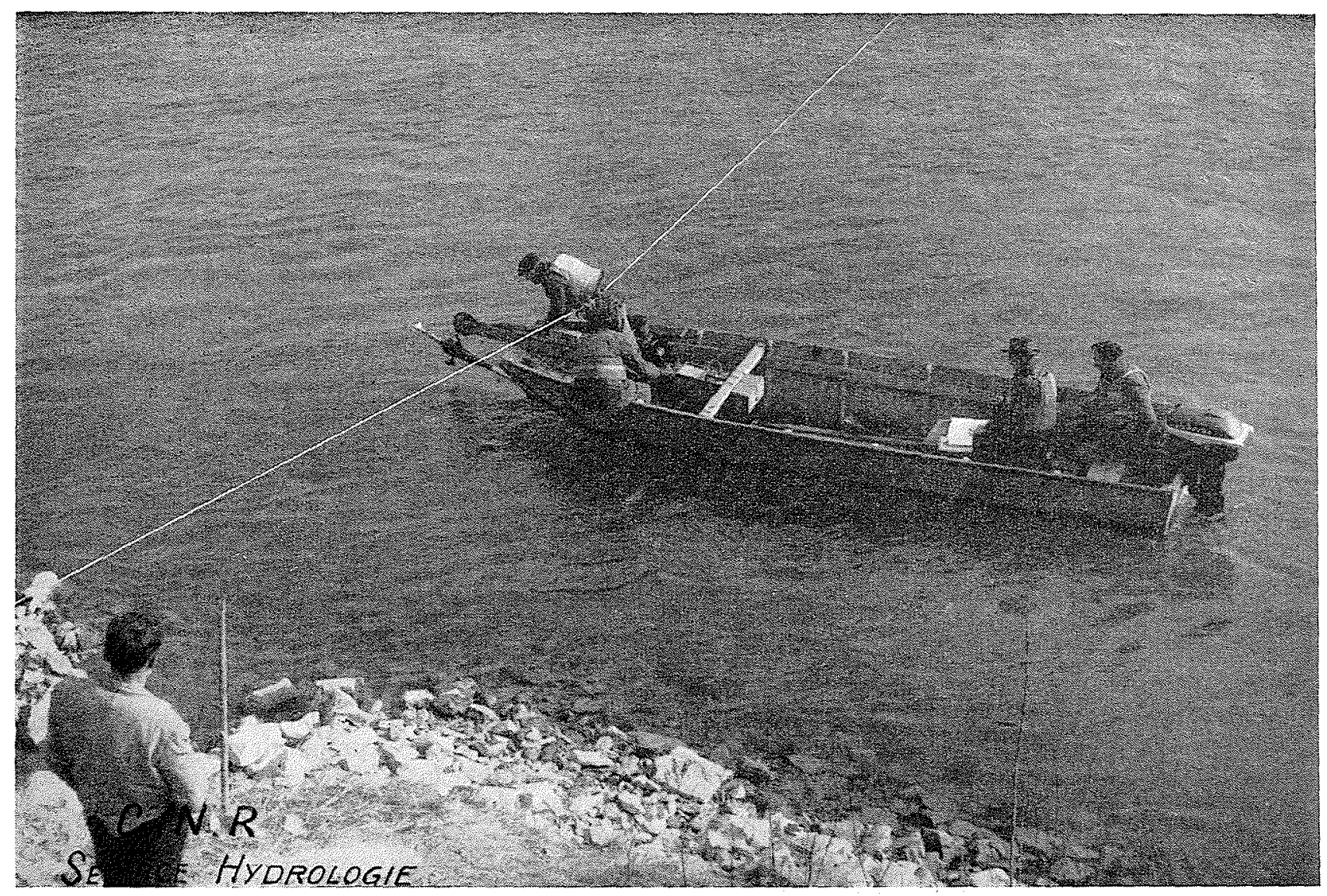

Fig. 2

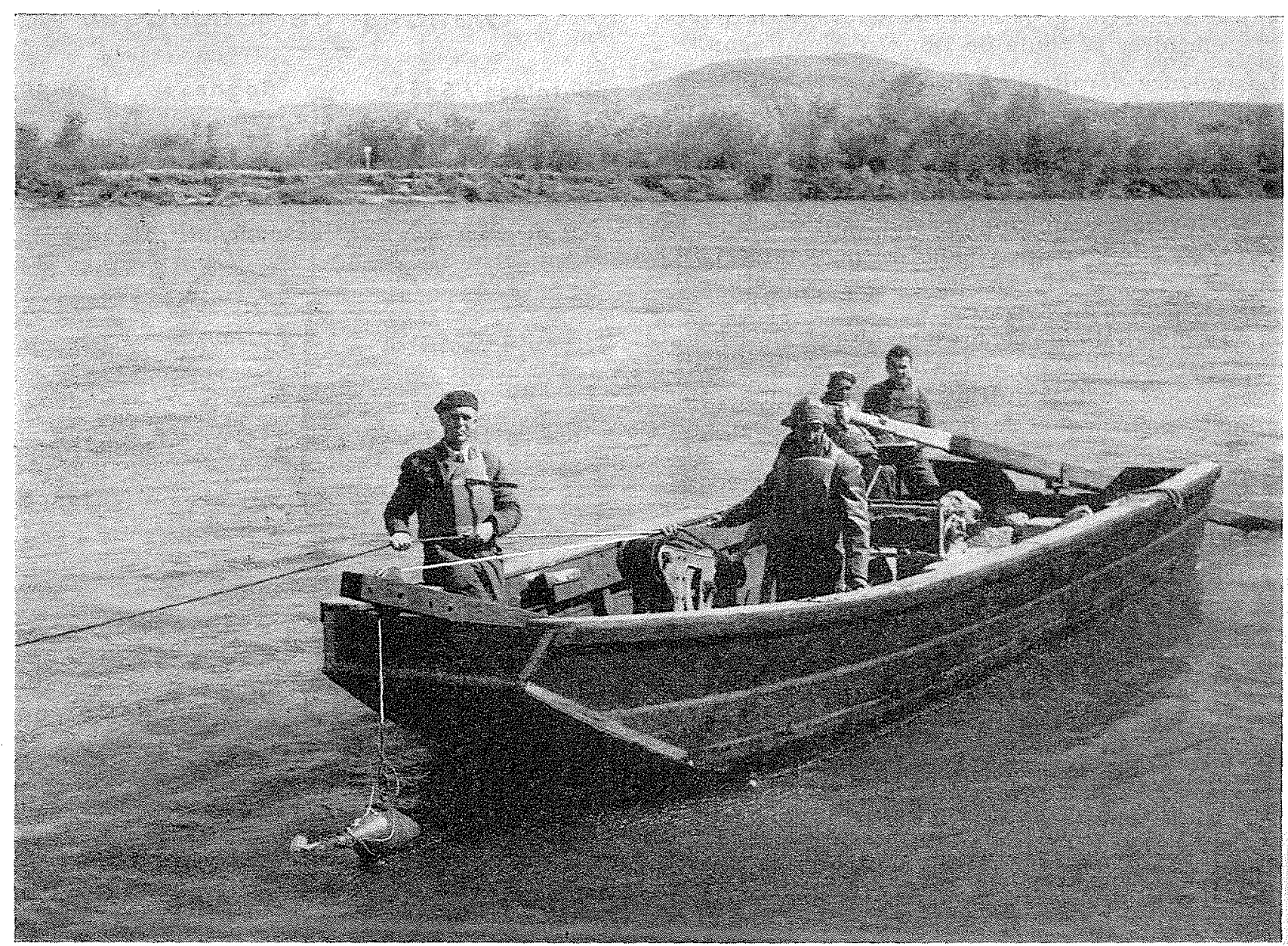

FrG. 3 
Deux stations : Sault-Brénaz et Bognes (Génissiat-aval), avaient été rétablies en 1949.

La cadence d'installation devait être en moyenne d'une station par an jusqu'en 1959; c'est donc une douzaine de stations de jaugeages que nous exploitons sur le Rhône.

Dans l'ensemble, l'installation est nautique: c'est à partir d'une barque qui se traille sur un câble que nous opérons. Les dimensions de la barque varient de $6 \mathrm{~m} \times 1,5$ à $10 \mathrm{~m} \times 3$ suivant la station. Le câble est soit fixe sur pylônes, soit traversé à la demande pour faire l'opération. (Fig. 2 et 3 .)
Trois installations sont téléfériques : Bognes, Châteaufort et la Balme.

Du point de vue précision, le dispositif nautique est préférable; toutefois, et surtout en temps de crue, il peut devenir assez sportif. Aussi, il nous arrive de limiter l'exploitation en téléf: rique pour les hautes eaux et de nous équiper en nautique pour les débits inférieurs.

Toutes les mesures sont faites à l'aide d'un moulinet suspendu lesté d'un saumon dont le poids varie de 50 à $150 \mathrm{~kg}$ suivant l'état du fleuve.

\section{III. - LES RÉSULTATS OBTENUS}

Parmi ces résultats : celui du fruit de notre expérience pour l'exploitation des stations ou l'exécution des jaugeages,

$1^{\circ}$ Il est souhaitable que la même équipe de jaugeages exécute les opérations à toutes les stations; c'est assurer une continuité dans les mé- thodes, favoriser l'entretien du matériel et donner de l'intérêt à la discussion des résultats;

$2^{\circ} \mathrm{Ne}$ jamais opérer avec un seul moulinet, mais disposer de plusieurs appareils qui seront utilisés dans une même journée;

$3^{\circ}$ Disposer d'un limnigraphe à chaque station de jaugeage.

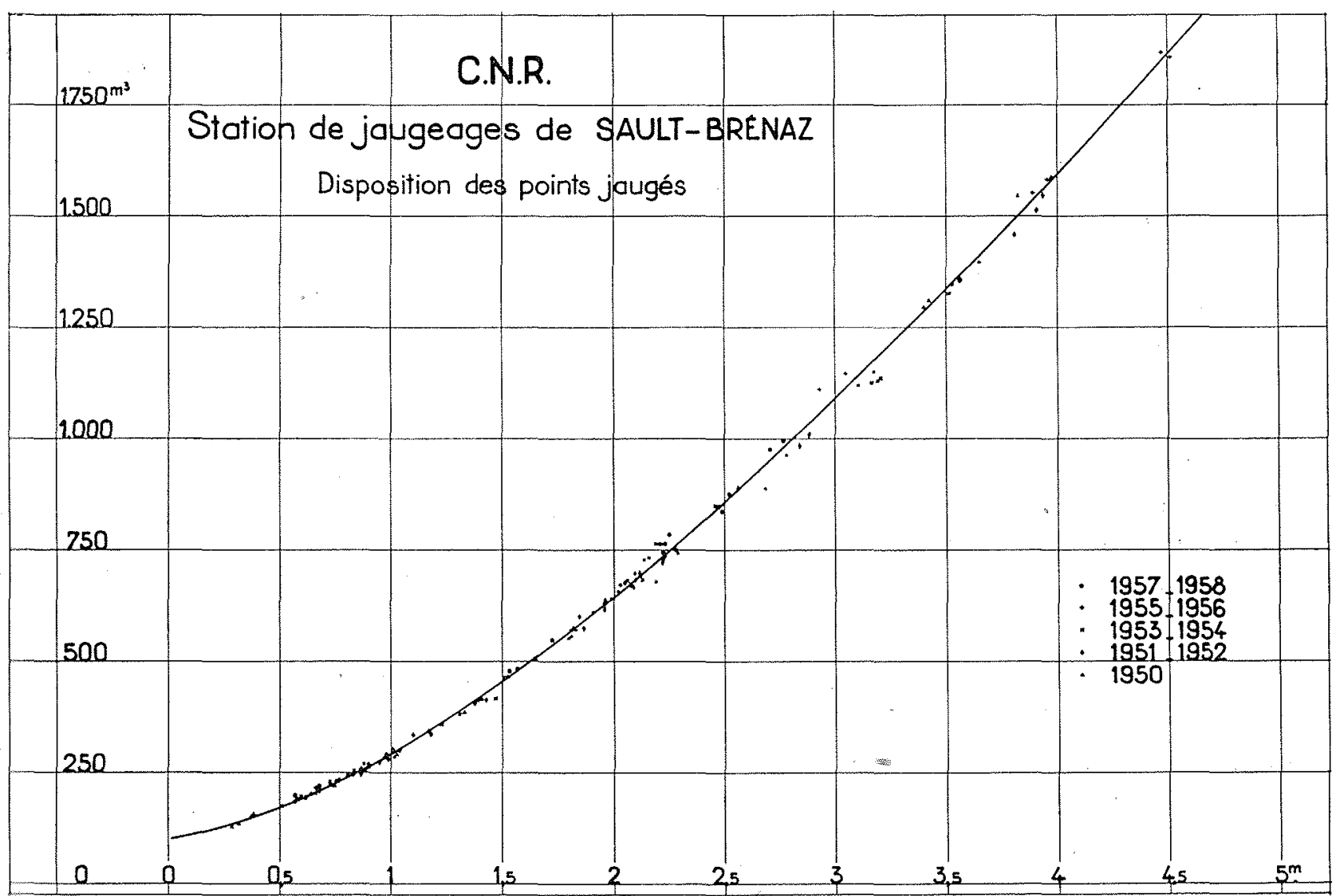

FIG. 4 


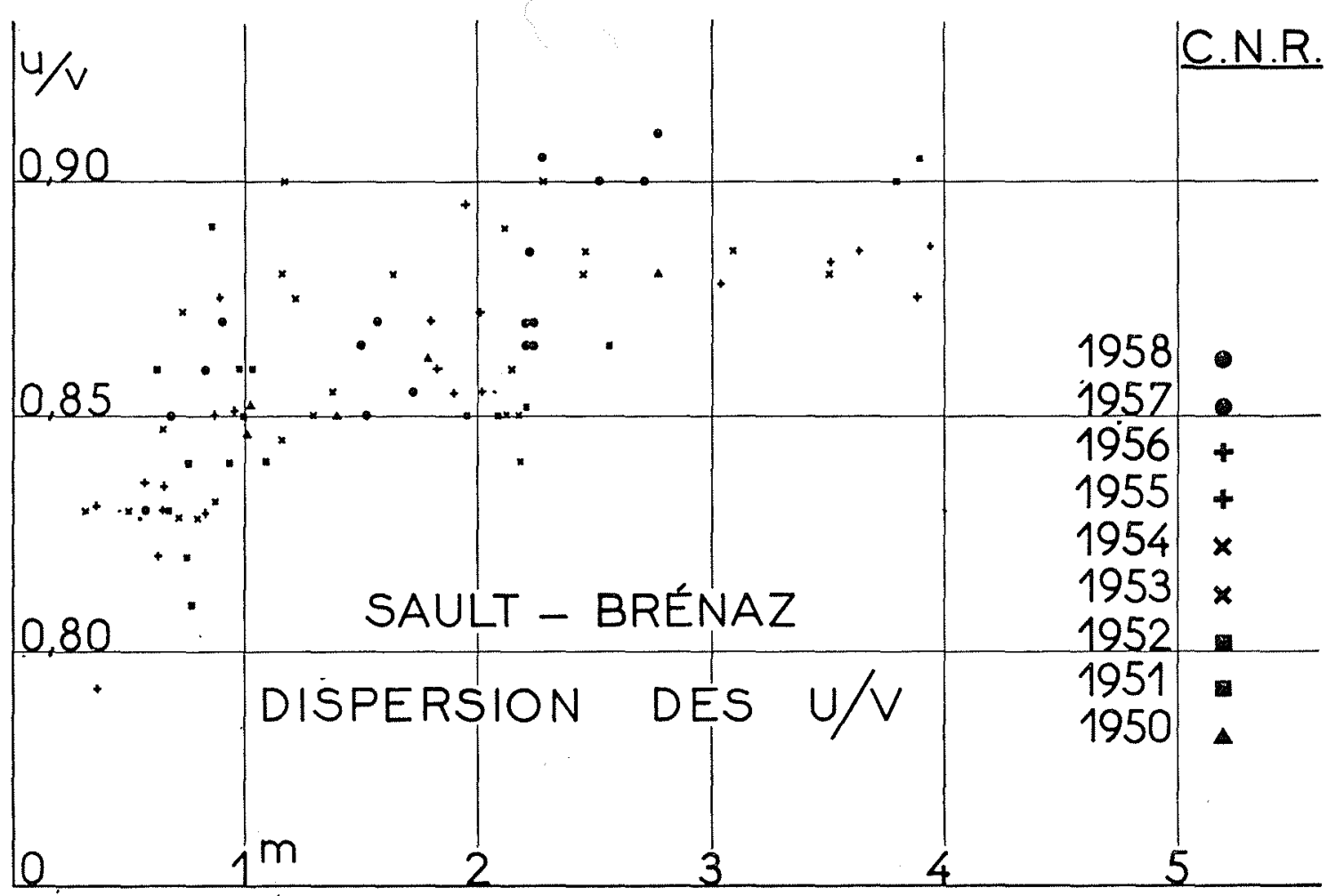

FIG. 5

Pour les résultats proprement dits des jaugeages, puisque je ne peux pas vous présenter ceux de toutes les stations et, pour ne pas paraitre tendancieux, je vous présenterai :

- La station de Sault-Brénaz qui est d'une stabilité remarquable;

- La station d'Oullins (Mulatière) dont le profil est médiocre;

- La station de Saint-Etienne des Sorts, où nous avons constaté des mouvements.

a) Station de Sault-Brénaz (fig. 4 et 5):

Cette station est remarquablement stable. Elle se situe sur le Haut-Rhône :

- A l'amont, la section du fleuve est en très faible pente et il n'y a pas de transit de débit solide;

- A l'aval, le lit du fleuve paraît stable aussi et il n'y a pas d'érosions.

Depuis 1950 nous y avons exécuté environ 150 jaugeages pour des débits variant de 130 à $2000 \mathrm{~m}^{3} / \mathrm{s}$.

Le diagramme (fig. 4) donne la position de ces points. Leur groupement est très satisfaisant et la courbe $H / Q$ est parfaitement définie.
Nous considérons cette station comme très bien étalonnée et nous nous bornons actuellement à exécuter chaque année trois ou quatre jaugeages pour des débits répartis sur l'étendue de la courbe, afin de s'assurer de la permanence de la stabilité de la station.

Toutefois, ce qui nous a troublés à cette station - comme à d'autres, mais surtout à celleci qui se présente dans de bonnes conditions -c'est la dispersion des $u / V$, comme vous pouvez le constater sur le diagramme (fig. 5).

\section{b) Station de la Mulatiene (fig. 6) :}

Pour l'établissement de cette station, nous avons utilisé les installations d'un ancien bac à traille et les conséquences s'en ressentent dans les résultats :

-_ le profil est trop triangulaire;

- les rampes de l'ancien bac troublent l'écoulement pour certains débits;

- les mouvements d'un banc de gravier amont se reproduisent dans le profil de la station;

- en crue, nous avons des débordements à l'aval qui influent sur les niveaux à la station;

- la situation des berges se prête mal à l'instal- 
lation d'un limnigraphe au droit de la station.

Les résultats obtenus figurés sur la courbe (fig. 6) font apparaître plus de dispersion qu’à Sault-Brenaz.

Certains d'entre vous penseront peut-être que ces résultats ne sont pas aussi mauvais que je voulais bien le dire. Il faut donc que j'ajoute que cette section du Rhône paraît être soumise à un creusement du lit très lent et que l'imparfaite sensibilité de la station nous gêne pour suivre cette évolution.

Cet exemple est néanmoins une occasion pour insister sur l'importance du choix de la station de jaugeages; c'est même à mon sens le problème le plus difficile et le plus important.

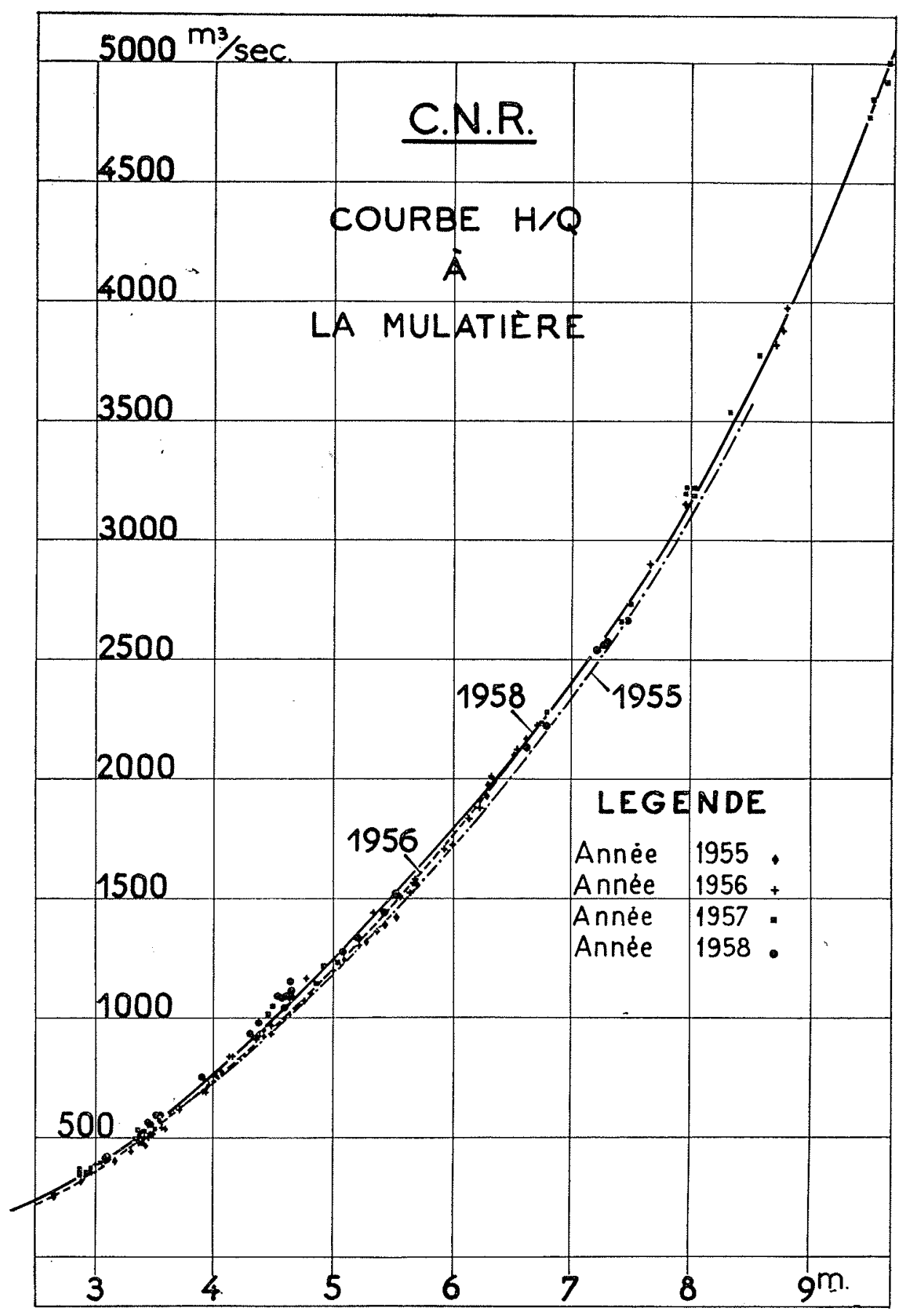

FIG. 6 
c) Station de SaINT-ETIENnE-des-Sorts (fig. 7) :

Elle est installée dans une section du Rhône où le lit du fleuve s'exhaussait certainement, mais Dieu seul sait dans quelles conditions exactes.

La restitution de notre aménagement de Donzère-Mondragon se situe maintenant 3 kilomètres plus à l'amont et il était bien évident que, sur près de 30 kilomètres de Rhône court-circuité, le changement de l'écoulement allait pro- fondément perturber le cheminement du débit solide.

On pouvait craindre un renversement de tendance et avoir un creusement du lit à la restitution au lieu de l'exhaussement du lit naturel. Je dis craindre, car, quelques kilomètres à l'aval, une barre rocheuse assez faiblement recouverte d'alluvions traverse le fleuve; un abaissement du lit pouvait la découvrir et la rendre dangereuse pour la navigation.

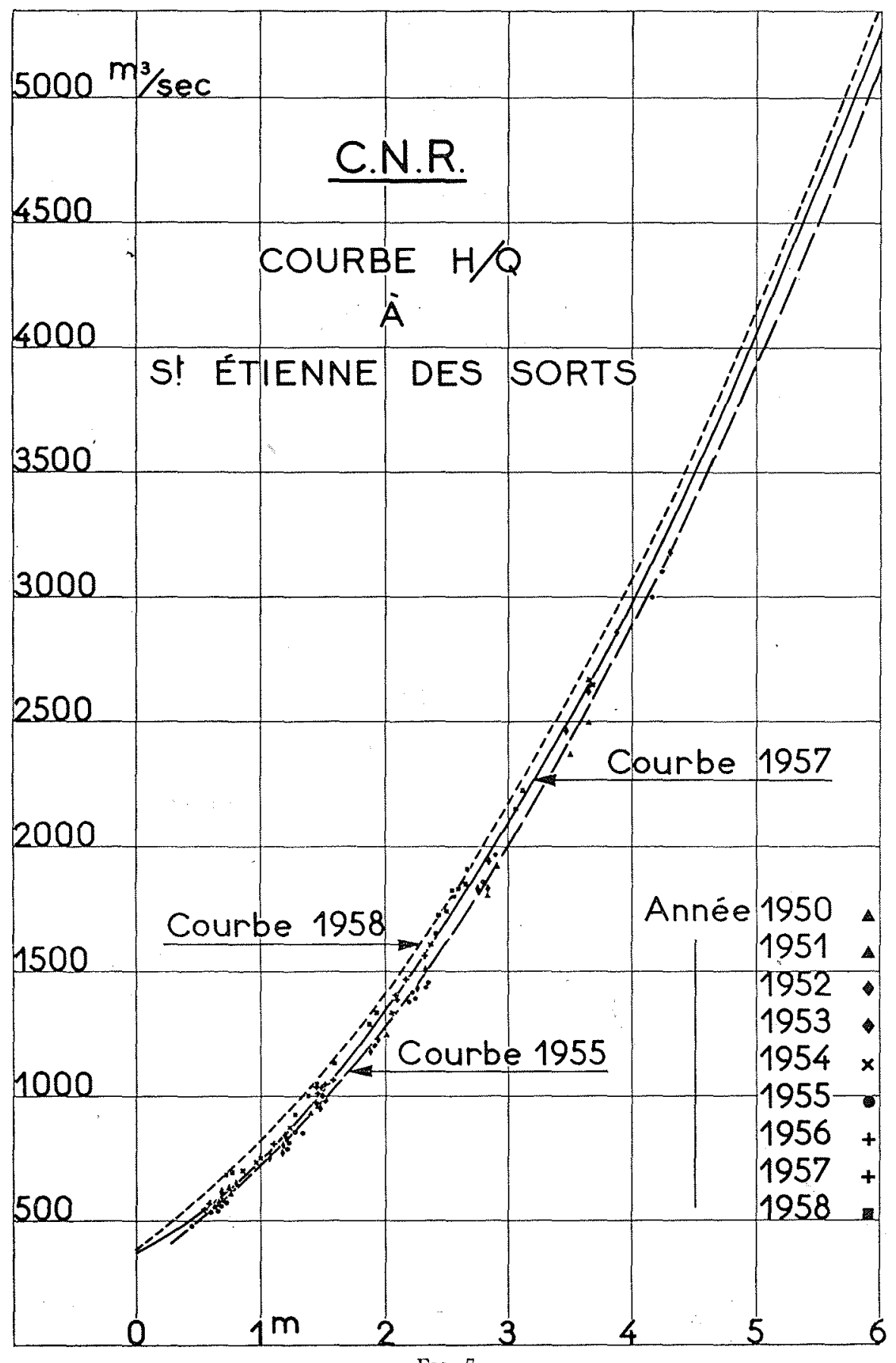

FIG. 7 


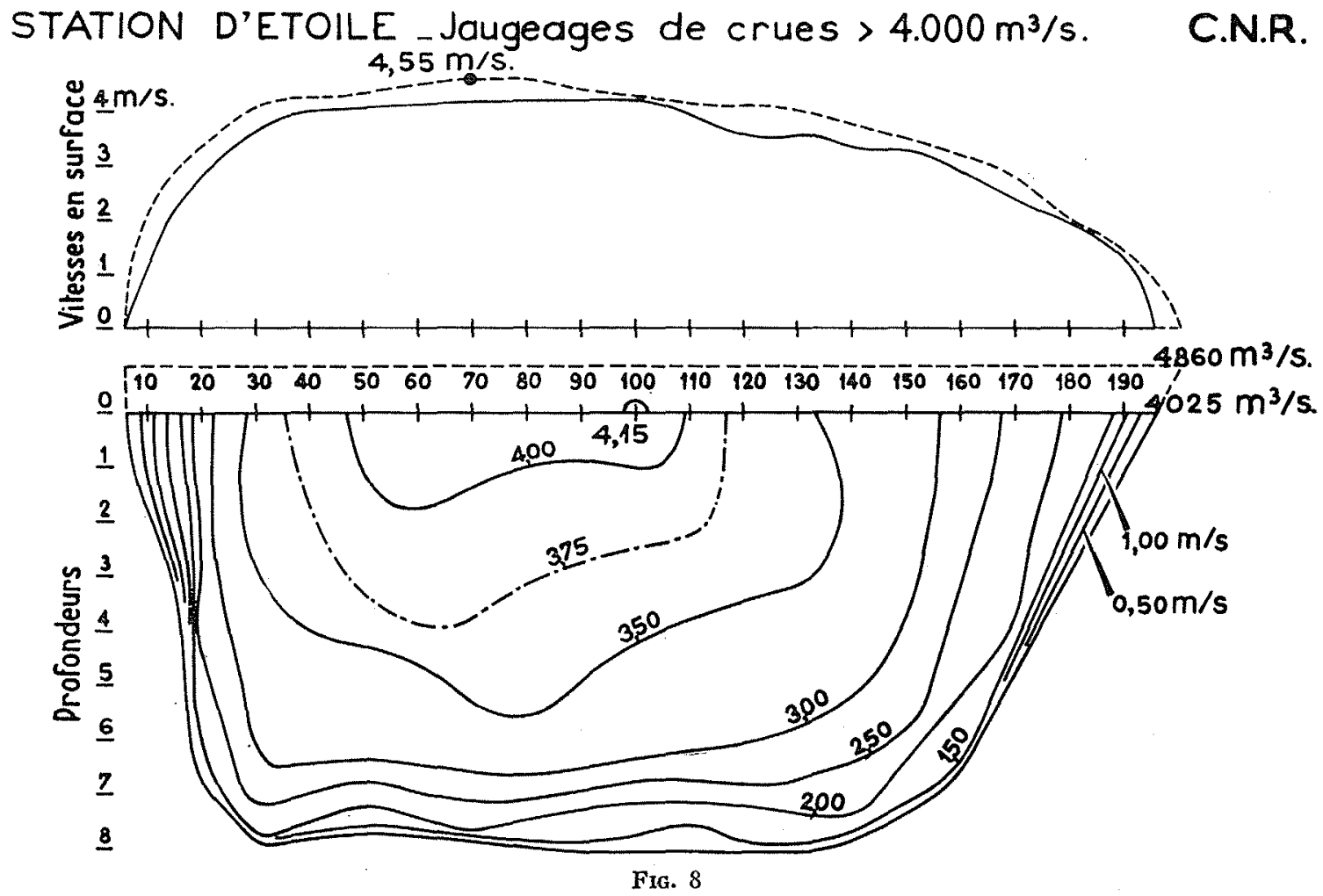

Le diagramme (fig. 7) montre les résultats obtenus. On assiste bien à un approfondissement du lit, mais avec ces constatations rassurantes : - D'une part, des périodes de forte hydrauli- cité, de crues - c'est le cas de l'année 1955 - provoquent un retour vers la situation initiale;

_- D'autre part, l'évolution apparaît lente.

\section{IV. - JAUGEAGES DES CRUES}

II n'est pas permis d'exposer les résultats d'observations hydrométriques sans parler des jaugeages de crues.

Bien que nous ayons saisi toutes les occasions, nous n'avons pas encore fait beaucoup de jaugeages de crue à chaque station.

Nous avons cependant mesuré les maxima suivants :

\section{A Sault-Brénaz :}

En surface $1870 \mathrm{~m}^{3} / \mathrm{s}$ soit $78 \%$ du débit estimé de la plus forte crue connue;

En toutes profondeurs $1515 \mathrm{~m}^{3} / \mathrm{s}$, soit $63 \%$ de cette même crue.

\section{A ra Mulatióre:}

En surface $5000 \mathrm{~m}^{3} / \mathrm{s}$ soit $82 \%$ du débit estimé de la plus forte crue connue;

En toutes profondeurs $3000 \mathrm{~m}^{3} / \mathrm{s}$ soit $50 \%$ de cette même crue.

\section{A ÉTOILE:}

En surface $4860 \mathrm{~m} / 3 / \mathrm{s}$ soit $60 \%$ du débit estimé de la plus forte crue connue;

En toutes profondeurs $4025 \mathrm{~m}^{3} / \mathrm{s}$, soit $50 \%$ de cette même crue.

Le profil de la figure 8 donne le résultat de ces deux dernières opérations: vous constatez que la profondeur d'eau atteint 8 mètres et que les vitesses dépassent $4 \mathrm{~m} / \mathrm{s}$; cela pour des opérations effectuées sur une barque malgré les difficultés résultant de la présence de corps flottants et des accès rendus difficiles par les submersions.

Je répèterai aussi que nous estimons qu'une des difficultés d'interprétation des mesures est le choix du $u / V$ lorsque les mesures ne sont faites qu'en surface. 


\section{V. - LE BENÉFICE DES OBSERVATIONS ET LES ETUDES EN COURS}

Les études proprement dites basées sur ces résultats ont été encore assez peu nombreuses.

Les renseignements recueillis n'en ont pas moins été très profitables :

- Pour la définition des ouvrages de nos aménagements hydro-électriques qui, depuis Donzère-Mondragon, ont fleuri sur le Rhône.
C'est ainsi que, par rapport à Donzère, nous avons pu gagner une passe sur le débouché du barrage de retenue aux aménagements amont, du fait d'une meilleure appréciation des débits des crues centenaire et millénaire;

- Pour les essais sur modèle réduit. Je crois savoir que les données fournies sont jugées

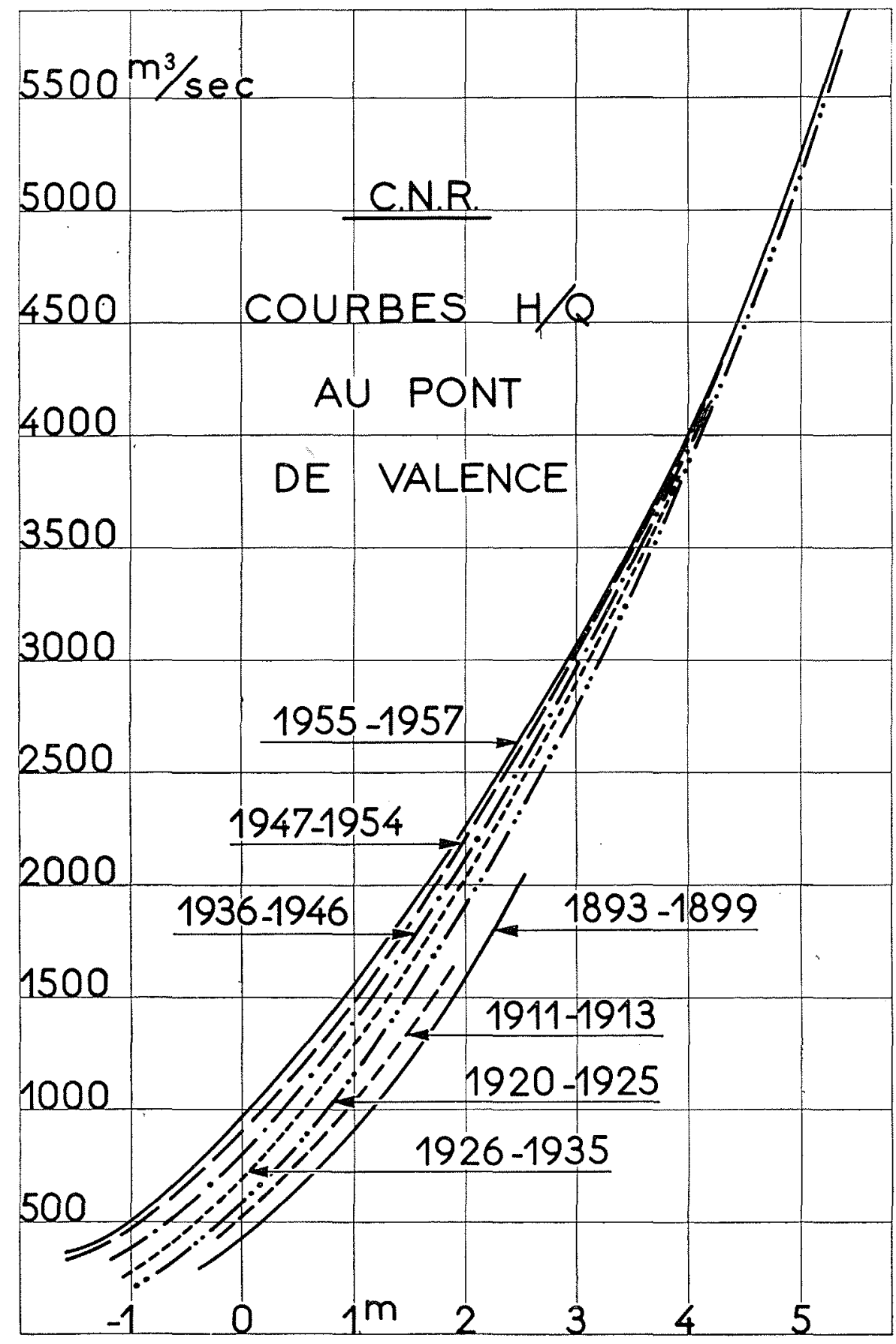

FIG. 9 


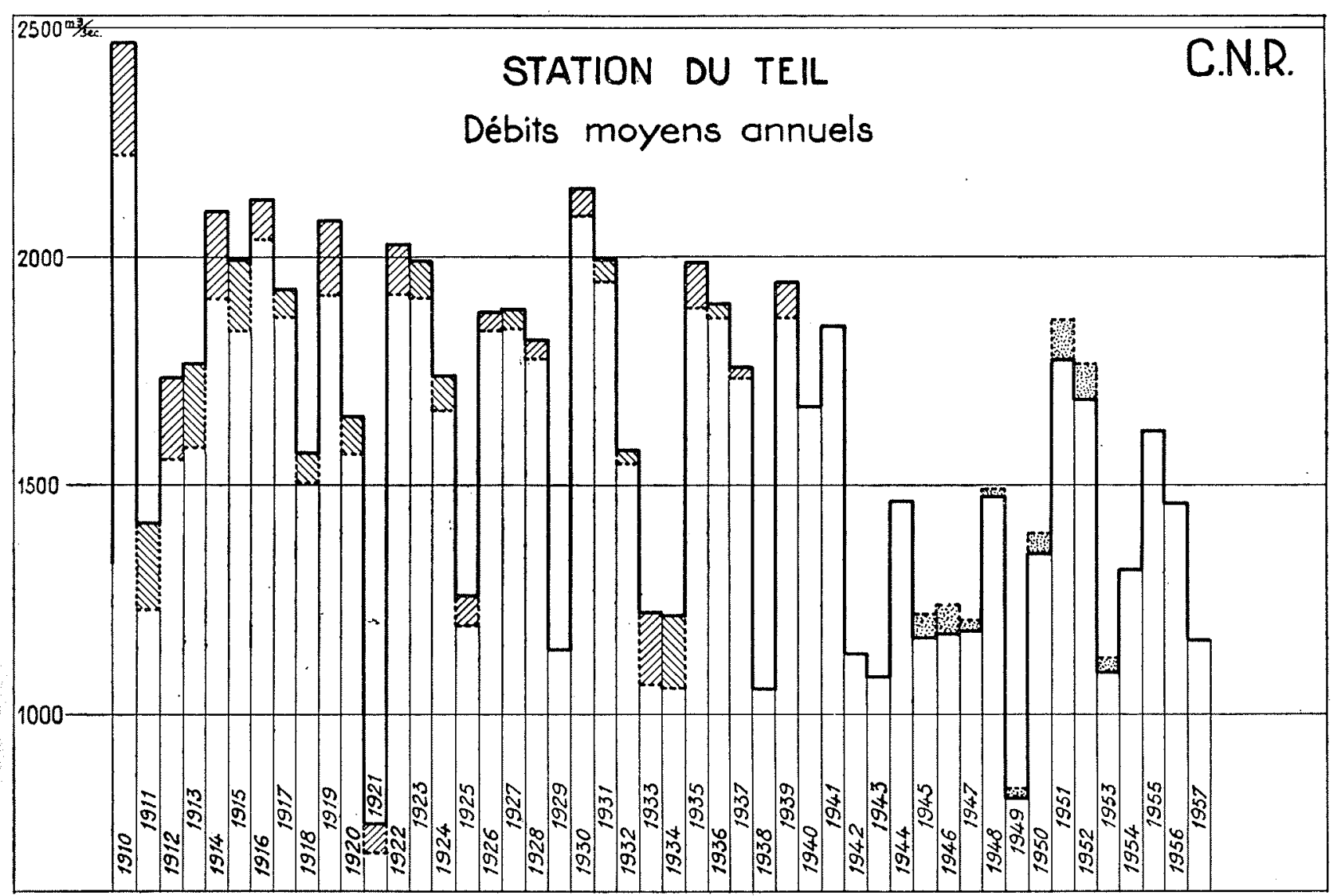

Fig. 10

assez satisfaisantes, bien que je comprenne qu'en ce domaine l'expérimentateur n'en ait jamais trop;

- Pour le débit des affluents. Je citerai spécialement la Saône, dont les débits étaient très mal connus; je dirais même absolument pas connus pour les faibles débits, car, sur une rivière canalisée, il n'y a pas de relation univoque entre le débit de la rivière et la cote à une échelle donnée. Par comparaison des débits fournis par les limnigraphes qui encadrent le confluent, nous pouvons très bien suivre les débits propres de la Saône;

- Pour les prévisions de débits. En ce qui concerne les crues, la connaissance des débits à chacune des stations successives et celle des temps de propagation permet de mieux suivre les crues dans le mécanisme même de leur formation et de leur transmission.

La question délicate des écrêtements de débits on fonction de la forme de l'onde de crue ou en conction des débordements a pu être étudiée de tucon assez précise.

La prévision des débits n'est pas seulement limitée aux crues mais est étendue à tous les débits. C'est ainsi que nous établissons une prévision hebdomadaire... à titre expérimental.. qui est suivie avec beaucoup d'intérêt par nos chantiers dans la vallée du Rhône, par la navigation et qui pourrait être utile pour les services de Mouvements d'Energie.

En ce qui concerne les débits moyens du fleuve, toutes ces mesures nous ont fait davantage prendre conscience de l'importance de la variation des courbes $\mathrm{H} / \mathrm{Q}$ dans le temps sur un fleuve à fond mobile. Nous sommes en train d'essayer de préciser cette évolution à certaines stations en reprenant les jaugeages au flotteur effectués dans le passé par le Service Spécial du Rhône.

C'est ainsi que, pour Valence, nous avons défini les variations de la relation $\mathrm{H} / \mathrm{Q}$ depuis 1893 (diagramme 9).

Au Teil, nous avons des bases moins complètes; néanmoins nous avons repris, en fonction des relations $\mathrm{H} / \mathrm{Q}$ les plus plausibles, le calcul des débits moyens journaliérs et des débits moyens annuels. Les résultats aplanissent beaucoup les difficultés d'un problème qui se posait sur la réduction progressive des débits annuels à cette station (fig. 10). 


\section{CONCLUSIONS}

Comme je vous l'avais laissé entendre au début de cette communication, nous n'avons jamais eu l'impression, au cours de ces dix années d'opérations hydrologiques sur le Rhône, de faire des choses transcendantes.

Telle n'était d'ailleurs pas notre intention.
Mais par une succession d'opérations méthodiques, un travail appliqué et persévérant, nous avons voulu rassembler les documents qui serviront de bases, des bases bien fondées, à des études ultérieures.

DISCUSSION

Président: M. Duffaut

M. le Président remercie M. Mauchamp de sa très intéressante communication et donne la parole à $M$. Marc Henry. Ce dernier fait un exposé complémentaire qui est résumé ci-après :

M. Marc Henny fait remarquer que les mesures relatées n'ont d'intérêt que dans la mesure où il existe, au moins en quelques sections du Rhône, une fonction bauteurs-débits.

En fait cette fonction n'est pas une fonction univoque du débit car l'évolution du lit impose une tendance; par ailleurs, si on exprime dans.une section donnée, la hauteur $\mathrm{H}$ en fonction du débit $q$ et du temps $t$, on constate qu'il existe des écarts autour d'une valeur moyenne; la fonction $\mathrm{H}(\mathrm{Q} t)$ est done une fonction aléatoire.

Sur un cours d'eau tel que la Drôme, dont les crues sont très rapides, la configuration du lit présente de grandes variations dans les régions où le lit n'est pas fixé :

- A la fin de chaque crue, le transport du gravier s'arrête brusquement, et ce gravier se dépose là où il est, ce qui donne au lit majeur un relief absolument chaotique. Dans ce lit majeur, les basses eaux se fraient un chenal qui n'a aucun rapport avec celui de l'étiage précédent;

- Lors de la crue suivante, les courants sont d'abord orientés par le lit mineur qui s'est formé en étiage, puis ils remplissent le lit majeur en détruisant la configuration du lit minenr.

Dans les régions où le lit n'est pas fixé, l'écoulement est en général torrentiel, et chaque obstacle produit un ressaut.

Dans une section donnée et pour un débit donné, la hauteur peut varier énormément suivant qu'il existe un ressaut juste en amont de la section (écoulement fluvial) ou juste en aval (écoulement torrentiel).

Les changements de configuration du lit, dont l'effet est fortement exagéré par. les passages de part et d'autre de Ia hauteur critique, font que la dispersion de la courbe des hauteurs-débits est énorme : on a constaté, sur modèle réduit, qu'on peut avoir pour $800 \mathrm{~m}^{3} / \mathrm{s}$ un plan d'eau plus élevé que pour $1200 \mathrm{~m}^{3} / \mathrm{s}$.

Sur le Rhône non aménagé, on constatait et on constate des phénomènes analogues, quoique avec une ampleur moindre, du fait que les crues sont beaucoup plus longues.

Sur le Rhône aménagé à courant libre, le lit est en rartie fixé, et les variations sont encore plus faibles. Au surplus, quand elles se produisent, l'action des eaux inoyennes tend à les effacer.
C'est ainsi qu'après les crues de l'hiver 1954-1955 le seuil d'Etoile, situé en aval de Valence, était exhaussé d'un mètre, mais après trois mois d'eaux moyennes, ce senil a repris son niveau antérieur.

Même lorsque le lit est bien fixé, ce qui est le cas à Sault-Brénar, il se produit des fluctuations dans le mode a'écoulement : pour un débit donné, la dispersion relative $d u / u$ de la vitesse moyenne $u$ est faible, et il en est de même pour celle $d v / v$ de la vitesse superficielle moyenne $v$; par contre, ainsi que nous l'a montré ii. Mauchamp, la dispersion relative du rapport $u / v$, soit :

$$
\frac{d(u / v)}{u / v}=\frac{d u}{u}-\frac{d v}{v}
$$

sans ètre bien grande en valeur absolue ( $\pm 4 \%$ ), est appréciable, ce qui parâit impliquer une haute probabilité pour que les ecarts $d u$ et $d v$ soient de sens inverse. Ouoi qu'il en soit, la dispersion des courbes de hauteurs-débits est acceptable sur le Rhône non aménagé, ot faible sur le Rhône aménagé, du moins pour les débits moyens et faibles qui sont contenus dans le lit mineur. Le lit majeur" est beancoup moins fixe et il peut s'ouvrir en crue un bras qui change l'écoulement, de sorte que la dispersion est beaucoup plus forte en crue.

Ceci étant de la dispersion, qu'en est-il de la tendance?

Sur le Haut-Rhône, les apports des affluents alpestres ct jurassiens se sont accumulés sur deux cônes de déjection : un dans la plaine de Chautagne, en amont du lac du Bourget, et un à $30 \mathrm{~km}$ en amont de Sault-Brénaz, dans lequel venait s'arrêter tout le débit solide grossier.

Les ouvrages ont quelque peu modifié la situation :

-.. C'est ainsi que Chancy-Pougny et Verbois ont donné lieu à une érosion du lit en aval; grâce aux chasses, cette érosion n'a pas fait place à un engraissement depuis la mise en eau de Génissiat.

- Depuis cette mise en eau, le gravier est arrêté a Génissiat et le lit paraît s'enfoncer dans le cône de déjection de Chautagne; les produits de l'érosion correspondante viennent s'arrêter sur le cône de déjection situé en amont de Sault-Brénaz, qui continue ì s'engraisser légèrement.

Dans le Bas-Rhône canalisé, il n'existe guère do digues insubmersibles en dehors du secteur aménagé par la Compagnie Nationale du Rhône. Les dépôts de limon qui se produisent en crue dans le lit majeur exhaussent le champ d'inondation; le développement de la végéta- 
tion, en favorisant le dépôt du limon, accélère cet exhaussement; enfin les constructions et ouvrages de toute nature entravent l'écoulement dans le lit majeur.

Pour un débit de crue donné, la part du débit qui est transportée par le lit mineur va done en augmentant d'une crue à la suivante, de sorte que la pente du lit mineur a tendance à diminuer.

C'est bien ce qu'on constate sur le Bas-Rhône canalisé, en aval du confluent de l'Isère, et cet effet est accéléré par le fait que les galets de l'Isère ne parviennent plus jusqu'au confluent : c'est ainsi que, depuis une centaine d'années, le lit s'est approfondi d'un mètre à Valence, ot s'est exhaussé en aval de Bourg-Saint-Andéol. Depuis que le débit solide est arrêté en presque totalité par les ouvrases de Donzere-Mondragon, le lit mineur s'abaisse en aval de ces ouvrages.

Par contre, en amont de l'Isère, la canalisation a probablement laisse un lit un peu large, de sorte que le pouvoir de transport du Rhône est nul : c'est dans cette région qu'on rencontre de nombreux seuils qui entravent la navigation. Mais cette région est soumise à crosion régressive en raison de l'abaissement du lit au confluent de l'Isère : actuellement cette érosion, qui a creusé le lit de $1,40 \mathrm{~m}$ en un siècle au droit de Tainlournon, se fait sentir jusqu'à Saint-Vallier.

Le déhit solide qu'on constate à Donzère, et qui a donné lieu à une étude présentée en 1955 avec M. Gemaenung, vient de l'érosion du lit entre SaintVallier et Donzère, ainsi que, pour une taible part, des alfluents.

Ailleurs les changements survenus paraissent résulter du fait de l'homme:

- En amont de Lyon, la concentration des eaux dans le bras de Miribel a donné lieu à un abaissement notable de la pente et à un accroissement considérable du pouvoir d'érosion; c'est ainsi que le lit s'est enfoncé en amont de Miribel, l'érosion régressive ayant abaissé le confluent de l'Ain d'un mètre. L'évacuation des apports n'étant pas assurée a l'extrémité aval du canal, ces apports ont formé un cône de déjection juste en amont de Lyon;

A la traversée de Lyon, les extractions de gravier provoquent un abaissement continu du lit.

M. Acrert, très intéressé par les deux exposés qui vemnent d'ètre faits, sugrère le rapprochement éventue Jo la courbe hauteur-débit avec lobservation directe 16. l'évolution des fonds et demande quel est le mode utvaluation des débits de la Saône, mentionnès par NI. Mauchamp.

Sur le premier point, M. HeNry indique que l'établiswiment d'un plan coté complet du fond, comme en faitit autrefois le Service spécial du Rhône, demande thancoup de temps, et que la Compagnie se borne en tonctal à dresser des profils en travers. Pour un certain Tombre de sections, la Compagnie calcule le coefficient n. de la formule $H=Q^{m}$, la hąuteur $H$ étant mesurée urwdessus du plus haut des points bas des seuils situés 41 aval.

Gur le deuxième point, M. HeNry répond que les faibles 4t moyens débits de la Saône ne sont comnus que par Vhorence entre les débits du Bas-Rhône et ceux du Thư Rhône. M. Mauchamp précise que des jaugeages twut exécutés en Saône lorsque les barrages sont ouverts Tr frand; ces mesures directes procurent un contrôle 16. debits obtenus par différence.

A. Aunfint reprend la question de la courbe univoque (11) non-univoque de la relation hauteur-débit, amorcéc 13\% M. Henny : cette relation dépend-elle de la pente ruerficielle? Or, M. Mauchamp a essayé d'opérer entre 1.ruk zones, c'est-à-dire à un endroit où le débit ne soit 11. an augmentation, ni en diminution. Il semble donc The, sur les biefs du Rhône oì la pente est faible, il y ait, comme sur tous les cours d'eau, une grosse différence, entre le débit de crue et le débit en décrue.

M. Henry répond que cette différence est incontestable et constitue une grosse préoccupation lors des jaugeages : ceux-ci ne sont en effet valables que s'ils sont exécutés en régime presque permanent, condition qui est loin d'être toujours observée en raison des variations des débits des usines situées en amont.

M. Mavchamp indique qu'en crue, il est généralement difficile d'opérer au cours de la montée, en raison des corps flottants; sur le Bas-Rhône, les décrues sont lentes, en raison des débits de la Saône, ce qui permet d'exécuter des jaugeages valables.

M. RÉméñ̌́ras demande si, pour améliorer les mesures en période de crue ou de décrue rapides, on a tenté de relier le débit $Q$ non seulement à la hauteur $H$ lue à l'échelle (par la classique « courbe de tarage» $Q(\mathrm{H})$, mais a la fois à cette hauteur et a la pente $i$ de la surface libre dans le secteur encadrant l'échelle (emploi d'une « surface de tarage $\%, Q=f(H, i))$; cela implique ia lecture d'au moins deux échelles assez éloignées pour déterminer correctement la pente $i$.

M. Mauchamp indique que quelques mesures de ce genre ont été faites sur la Saône en crue pour contrôler Jes débits de cette rivière, donnés par différence, comme indiqué plus haut.

M. Hennx ajoute que ce calcul n'offre guère d'intérĉt sur la Saône, dont les débits varient très lentement (régime pseudo-permanent). Sur le Rhône, on n'est pas toujours en régime pseudo-permanent, notamment lors de certaines crues d'affluents qui peuvent passer de (i) à $3000 \mathrm{~m}^{3} / \mathrm{s}$ en une journée : l'application de la méthode de jaugeage à deux echelles y serait donc intéressante, mais l'erleur sur les pentes peut y être considérable.

M. RÉMÉnÉns remarque que, dans la plupart des cas, l'altération de la courbe de tarage (établie en régime permanent) en période de crue ou de décrut rapides résulte non pas de la « pente d'accélération $»(1 / g)(\delta Q / \delta t)$, mais de la modification des « conditions aux limites 》 aval (« contrôle $»$ aval) du bief contrôlé par l'échelle considérée, du fait de la propagation de la crue.

H. Henry estime que l'écrêtement peut être appréciable pour les crues rapides des afflents cévenols : des crues de l'Ardèche, de l'ordre de 4000 a $5000 \mathrm{~m}^{3} / \mathrm{s}$ sont survenues alors que le Rhône, au droit de la dérivation de Donzère-Mondragon, ne débitait que quelques dizaines de $\mathrm{m}^{3} / \mathrm{s}$; c'est-à-dire que le lit du Rhône était pratiquement à sec.

Dans des cas de ce genre, il se forme en amont du confluent un lac qui s'étend sur 6 ou $8 \mathrm{~km}$; en aval, le plan d'eau du Rhône monte rapidement, il $y$ a donc unc accumulation importante dans ce lit, et il $y$ correspond un sérieux écrêtement du maximum. En aval de la restitution du canal de Donzère-Mondragon, la montée du plan d'eau se ralentit beaucoup, et l'effet d'écrêtement est moins intense.

M. Lugrez pose denx questions :

1. Dans le cas de l'usine de Génissiat, a-ton essayé de faire un recoupement entre les débits déterminés par les installations de l'usine et les débits mesurés en des stations de jaugeages voisines? Un contrôle permanent des débits turbinés par l'usine est-il possible par ce procédé?

20 Quels sont, daus le cas de Donzère, les éléments de la prévision des débits effectuée en utilisant les observations faites aux divers points de mesure, et à laquelle M. MAUCHAMP à fait allusion?

A la première question, M. Henry répond que, Génissiat étant une usine de pointe, les débits sont très 
variables à la station aval de Bognes, située entre Ciénissiat et le réservoir de compensation de Seyssel; de ce fait, les indications du limnigraphe de Bognes ne peuvent pas se traduire en débits avec une précision complète. Toutefois, la production d'énergie est en bon accord avec celle qu'on peut calculer à partir des débits.

A la deuxième question, MM. Henny et Mauchamp répondent que les prévisions immédiates de débit at Donzère sont faites à partir des données recueillies au Pouzin. La Compagnie s'efforce en outre d'établir des prévisions à plus longue échéance d’après les relevés de l'amont et les prévisions météorologiques : à titre expérimental, elle essaie de donner des prévisions hebdomadaires; le 18 janvier 1959, elle a pu indiquer que le débit à Donzère serait de $1500 \mathrm{~m}^{3} / \mathrm{s}$ au moins jusqu'à la fin du mois.

M. le Professeur Gumber. (Columbia University of New York) indique que les phénomènes saisonniers, tels qu'ils existent dans l'hydrologie fluviale peuvent être analysés a laide de la distribution circulaire normale. Cette méthode donne un critère pour la régularité de ces phénomènes. Elle pourrait être appliquée aussi à la courbe de jaugeage pour voir si elle est soumise à des influences saisonnières.

M. ANDRÉ demande à M. Maúchamp pour quelles raisons il considère les jaugeages effectués à partir de barques comme plus précis que les mesures faites au moyen d'un téléphérique.

M. Mauchamp répond que, sur un fleuve large, profond, et à courant vif, comme le Rhône, la poussée sur le moulinet et son câble de suspension, fait que ce câble s'incline fortement sur la verticale, ce qui fausse d'autant plus les mesures de profondeur que le câble porteur, en raison de la navigation, est très élevé au-dessus du plan d'eau. Sans doute peut-on utiliser un câble de retenue dont la traction est encaissée par une deuxième traille installée en amont, mais cela complique beaucoup l'installation.

M. ANDrÉ considère qu'en fait, étant données les oscillations du fleuve peu profond qu'est le Rhône, les mesures lui semblent plus faciles et plus sûres à partir d'un point fixe, indépendant des fluctuations, comme cela est le cas du téléphérique.

M. Mauchamp remarque que les mesures de profondeur sont faites avec beaucoup de précision : le périmètre mouillé est établi en très basses eaux au cours d'un sandage particulier et la section est, ensuite, seulement contrôlée an cours des observations : si ce contrôle révèle des variations très nettes, un nouveau sondage est pratiqué.

M. ANdré demande également si MM. Henry et MaycHaMp n'ont pas observé pendant les erues un creusement du lit suivi d'un remblaiement.

M. Henry suppose qu'il en est ainsi sur la Drome, mais non sur le Rhône.

M. Mauchamp précise que des mesures de profondeur, faites pendant les crues avec des contacts de fond spéciaux destinés à lutter contre la dérive, n'ont révélé aucun creusement ni remblaiement important sur le Rhône.

M. Tissier suggère à M. Mauchamp de donner à la S.H.F. la valeur corrigée des débits des stations importantes du Rhône, notamment de celles qui sont publiees dans l'Annuaire Hydrologique de la France; telle est par exemple la station du Teil dont il vient de présenter le graphique.

M. MaUchamp répond qu'il donnera ces débits rectifiés aussitôt qu'il aura pu les établir sur le Rhône à plusieurs stations et les corréler, c'est-à-dire dans quelque six mois, en vue de leur publication dans l'Annuaire 1958.

M. Tissien demande si l'on peut extrapoler aux très grandes crues (lorsque la rivière sort de son lit) l'augmentation du rapport $u / v$ avec le débit, le graphique d'ailleurs excellent présenté par M. Mauchamp semblant résulter de mesures effectuées sur la rivière restant dans son lit mineur.

M. MaUchamp répond qu'à la station de Sault-Brénaz, pour laquelle le graphique a été construit, il n'y a pas de débordement et que dans les cas de rivières débordantes, la courbe ne s'applique pas, mais la tendance a l'augmentation de $u / v$ ne fait que s'accentuer.

M. Gogombles demande si la C.N.R. a étudié le probième de la langue salée du Rhône inférieur (surtout du petit Rhône) et l'influence des prélèvements d'eau en amont sur la position de l'interface eau douce, eau salée.

M. Madchamp répond que cette étude n'est pas du ressort de la C.N.R., mais les Services des Ponts et Chaussées, qui ont le petit Rhône dans leurs attributions, et de l'Agriculture, qui s'occupent de la Crau, l'ont peutêtre réalisée.

M. Hanny confirme et précise la réponse de M. MaUCHaMP : la C.N.R. ne s'intéresse guère, au point de vue hydro-électrique, au Rhône en aval d'Arles, qui se trouve à $5 \mathrm{~m}$ au-dessus de la mer; elle sait cependant qu'il y a une difficulté et que cette difficulté augmente si le petit Rhône n'est pas convenablement alimenté. Il se pourrait qu'à la suite des prélèvements croissants sur Ic Rhône, la langue salée remonte dans le petit Rhône, pendant l'étiage. Pour que la proportion des eaux dans le grand Rhône et le petit Rhône ne se trouve pas changée d'une façon dangereuse, la C.N.R. a proposé d'éviter, dans le secteur qui la concerne, des prélèvements excessifs, surtout en étiage.

M. PARDÉ déclare qu'il aurait, sur la communication de MM. Henry et Mauchamp, une infinité de choses à dire et de questions à poser. Mais il veut simplement se borner à exprimer qu'il a rarement entendu, sur l'étude hydrométrique d'un fleuve, un exposé aussi intéressant, aussi riche en documentation nouvelle et posant autant de questions utiles.

M. le Président remercie $M$. PARDÉ de son intervention et associe l'assemblèe aux félicitations qu'il adresse aux auteurs. 